\title{
Rumor Propagation Model under Limited Information Exchange
}

\author{
Wu Mengyun* and Chen Wenjuan
}

Wu Mengyun and Chen Wenjuan are with the School of Finance \& Economics, Jiangsu University, Zhenjiang 212013 , P.R. China

\begin{abstract}
Rumor propagation has been well studied in the past decade, the main concentration is focused on the dynamic behavior analysis of model system, but little attention is paid to the limited information exchange among nodes in the network topology. In this paper, we numerically investigate the limited information transmission influence the rumor spreading. The information packet transmission quantity $m$ is introduced, which means how many messages can an individual send to his friends every time. Simulations illustrate that people transmit more messages to others, the rumor disappear sooner. In addition rumor propagation in homogeneous network has more influence than in inhomogeneous network.
\end{abstract}

Keywords: Information packet, inhomogeneous network, limited information exchange, rumor propagation.

\section{INTRODUCTION}

Rumors are part of our everyday life. People disseminate rumors in order to achieve the purpose of increasing awareness, slandering others, manufacturing momentum, diverting attention, causing panic and so on. The spread of rumors can shape the public opinion in a country, greatly impact financial markets and cause panic in a society during wars and epidemics outbreaks [1-4].

Rumors can be viewed as an "infection of the mind", and their spreading shows an interesting resemblance to that of epidemics. However, unlike epidemic spreading quantitative models andinvestigation rumor spreading dynamics have been rather limited. A classical model for the spread of rumor was introduced by Daley and Kendal $[5,6]$, and then many researchers have used the model extensively in the past for their quantitative studies [7-15]. Lv and Wang [16] stated that the spread of rumors, the public panic and the destruction of social order would interact with one another and could promote a proliferation of a significant emergency event. Zhang [17] proposed an interplay model between rumor spreading and emergency development and explained the different functions of rumor spreading at different stages of emergency development. Liu et al. [18] studied the case of a general network and found that the final percentage of population who heard the rumor decreases with a network structure parameter. Zhao et al. [19] analyzed the dynamics of rumor spreading on homogeneous networks considering the forgetting mechanism and concluded that the final state of stiflers depends greatly on the average degree of networks.

An important shortcoming of the above class of models is that they have not taken into account the limitations of the information exchange. They all assume that an individual could get information from all of his neighbors simultaneously. Obviously, this is not reality. For instance, although you have a lot of friends, you may not communicate with all of them at one time. On the other hand, you cannot receive all of your friends' information at a time step. Once an ignorant cannot get information from its neighbors who are spreaders, it could not become a infected. This factor has a great influence on the spread of rumors.

In this paper we introduce the information packet transmission quantity $m$, which means how many messages a node can send to its neighbors at one time. In our criterion, each message to be sent to its neighbors random. Under such condition, a node may transmit several messages or none to a neighbor node at a time step. Moreover, if an individual send more than one messages to a neighbor node at a time, it means the node would like to communicate with the neighbor node at this moment. When its neighbors could receive message from the node, the neighbors may change its state. Our model differs in three main respects with early work of Zhao [20]. Firstly, an individual only send a few messages to his neighbors at a time step is considered, then not all neighbors of the individual could receive information from it. Secondly, we assume a node status changes just depend on what information could it get. For example, although an ignorant connect with spreaders, he cannot become spreader if he could not get any information packet from those spreaders. We believe that the assumption is more reality in the real world. Finally, an comparison between ER random network and BA scale-free network was investigated.

This paper is organized as follows. In Section 2 a new rumor propagation model under limited information exchange was described. In Section 3 we present the numerical simulations on a homogenous network. In Section 4 the numerical simulations on an inhomogeneous network was illustrated. Finally some conclusions are given in Section 5. 


\section{MODEL DESCRIPTION AND EXPLANATION}

We divided the population into four groups: $I, S, H$, and $R$. Here $I, S, H$, and $R$ stand for the people who never heard rumor, those who are spreading rumor, those who coming from the spreaders due to forgetting mechanism and restart spreading the rumor by remembering, and the ones who heard rumor but do not spread it, respectively.

As we all known, at time $t$, one person won't send messages to all of his friends, on other words, one cannot get all his acquaintances' information at one time step. Furthermore, although a node may connect with many other nodes, its state cannot be changed if there are no any neighbors' information packets to it. Moreover, it has one old saying that three people spreading reports of a tiger make you believe there is one around. So an ignorants will become spreaders easily and become stiflers difficulty while they receive information from more spreaders. On other hand, the hibernator can be prone to awaken when more spreaders transmission information to him. Also, while spreader get more information from another spreaders or hibernators or stiflers, they may bore the rumor and think the rumor is wrong, so the probability that they become stiflers increase.

Base on above analysis, we improve previous works in [20]. In our new rumor propagation model, we suppose that at each time step, a node only can transmit $m$ information packets to its neighbors (each information packet randomly chooses a neighbor node arrive). If a neighbor nodecould receive more than one messages from the node at a time, that means the node would like to communicate with the neighbor node at this moment. On the other hand, a node could receive information packets from its several neighbors at time $t$, and $m_{I}(t), m_{S}(t), m_{H}(t)$ and $m_{R}(t)$, represent the number of ignorants, spreaders, hibernators, and stiflers who send messages to it, respectively. At each time step, some of them will change its state by collecting information from its neighbors.

The principle of our new model for rumor propagation under limited information exchange is as follows:

(i) When an ignorant can receive information from a spreader, the ignorant becomes a spreader with probability $P_{I S}=\lambda$, namely spreading rate, and if an ignorant can receive information from more than one spreaders at one time step, the ignorant becomes a spreader with probability $P_{I S}=1-(1-\lambda)^{m_{S}(t)}$. So we have

$$
P_{I S}= \begin{cases}0, & m_{s}=0 \\ 1-(1-\lambda)^{m_{S}(t)}, & m_{s} \geq 1\end{cases}
$$

(ii) When an ignorant can receive information from a spreader, the ignorant becomes a stifler with probability $P_{I F}=\beta$, namely refusing rate. Also, an ignorant will becomes a stifler with probability $P_{I F}=\beta^{m_{S}(t)}$, if he can receive information from many spreaders at time $t$. So we can get:

$$
P_{I F}= \begin{cases}0, & m_{s}=0 \\ \beta^{m_{s}(t)}, & m_{s} \geq 1\end{cases}
$$

(iii) The hibernators who come from the spreaders at a rate $\delta$ (forgetting rate) to reflect the forgetting mechanism. Hibernators spontaneously become spreaders at a rate $\xi$ (spontaneous remembering rate). When a hibernator receives information from a spreader, the former will become the latter with probability $\eta$, namely wakened remembering rate. Furthermore, when more spreaders sent messages to him, the hibernator will become latter with probability $P_{H S}=1-(1-\eta)^{m_{S}(t)}$. So we can get:

$$
P_{H S}= \begin{cases}0, & m_{s}=0 \\ 1-(1-\eta)^{m_{S}(t)}, & m_{s} \geq 1\end{cases}
$$

(iv) When a spreader obtain information from another spreaders or hibernators or stiflers, only the initiating spreader becomes a stifler at a probability $\alpha \frac{m_{S}(t)+m_{H}(t)+m_{R}(t)}{k}$, where $\alpha$ is positive constant means information sensitivity and the $k$ is the total number of its neighbors.

The refined rumor spreading process is shown in Fig. (1).

\section{RUMOR PROPAGATION IN HOMOGENOUS}

In this section, we study how information packet transmission quantity influences rumor spreading on a homogenous network. Without loss of generality, we consider an Erdos-Renyi(ER) network with $N=2000$ and average degree $\langle k\rangle=6$, which can be constructed by the algorithms given in $[21,22]$. In the initial condition, there is only one spreader in the network, and the others are ignorants.

We fix the parameters $\lambda=0.8, \beta=0.2, \alpha=0.3$, Fig. (2) illustrates the densities of the four groups in different state as a function of time, where a node could transmit information to all its neighbors. What can be seen from the simulation is that the number of spreaders rapid growth and then reach the peak and thereafter declines. The proportion of hibernator is similar to the spreader. Moreover, the number of ignorants and stifles rapid decrease and increase respectively.

Above all, if one can access to information from all his acquaintance, the rumor would disseminate faster and then disappear sooner. The next we will investigate rumor propagation model in social networks under limited information exchange.

First, we study the influence of information packages transmission quantity by a node on rumor spread at each time step. We fix the parameters $\lambda=0.8, \beta=0.2, \alpha=0.3$, $\delta=0.6, \xi=0.5, \eta=0.5$. Figs. (3 and 4) show the evolution of $S$ and $R$ for three typical $m=1,5$ and 10, respectively.

Fig. (3) show that the peak of $S$ increase with the increase of $m$, and the densities of spreader reach peak fast and rapid declines after that. On the other hand, with the decrease of $m$, the smaller of the maximum rumor influence, but the longer time it takes for the spreaders to decline to zero. Fig. (4) describes that densities of stiflers increase fast with more 


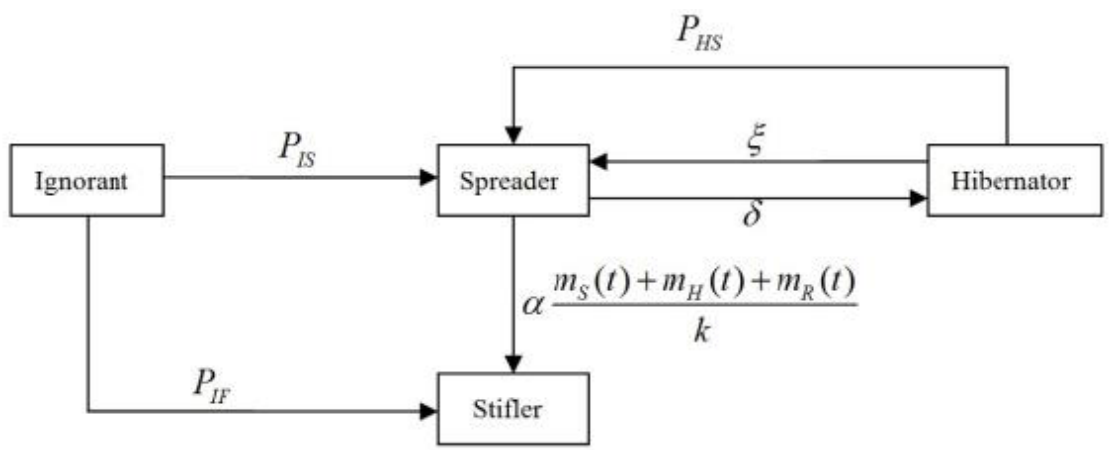

Fig. (1). Refined rumor spreading process.

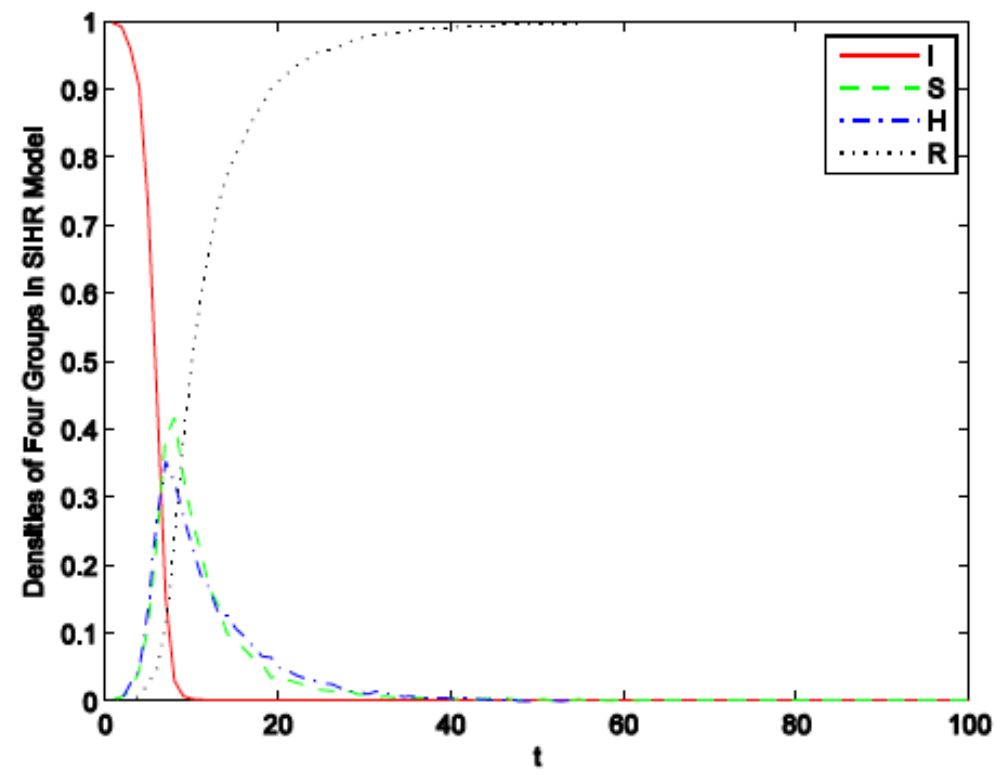

Fig. (2). Densities of Four Groups in homogeneous network.

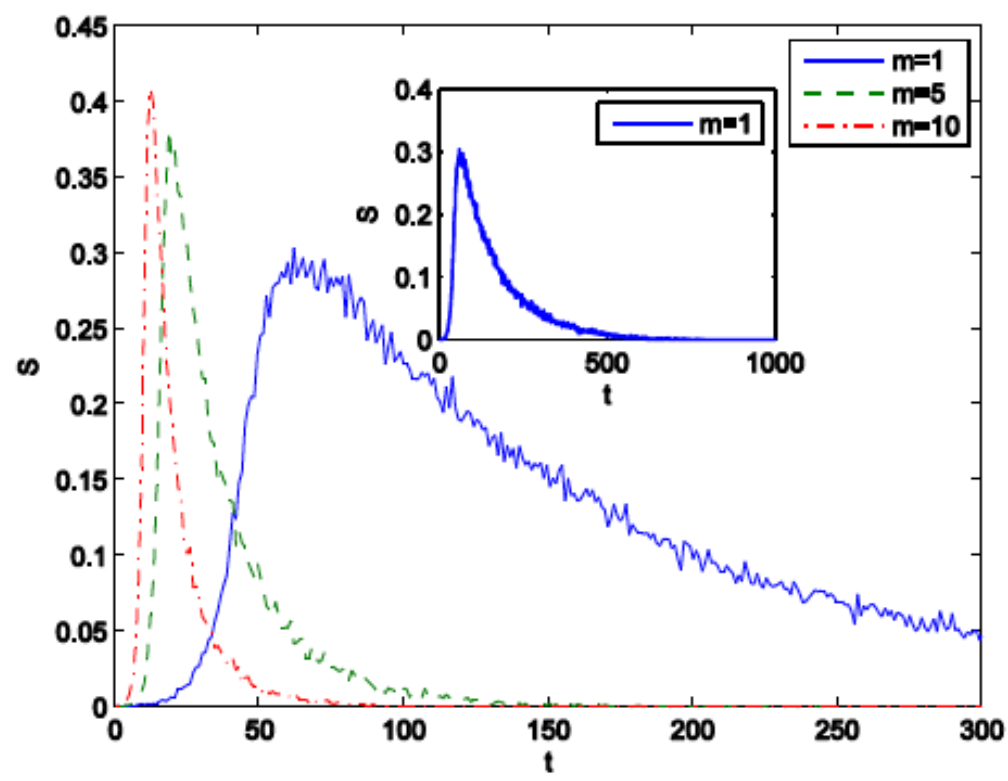

Fig. (3). Densities of spreaders over time with different information packet transmission quantity in homogeneous network. 


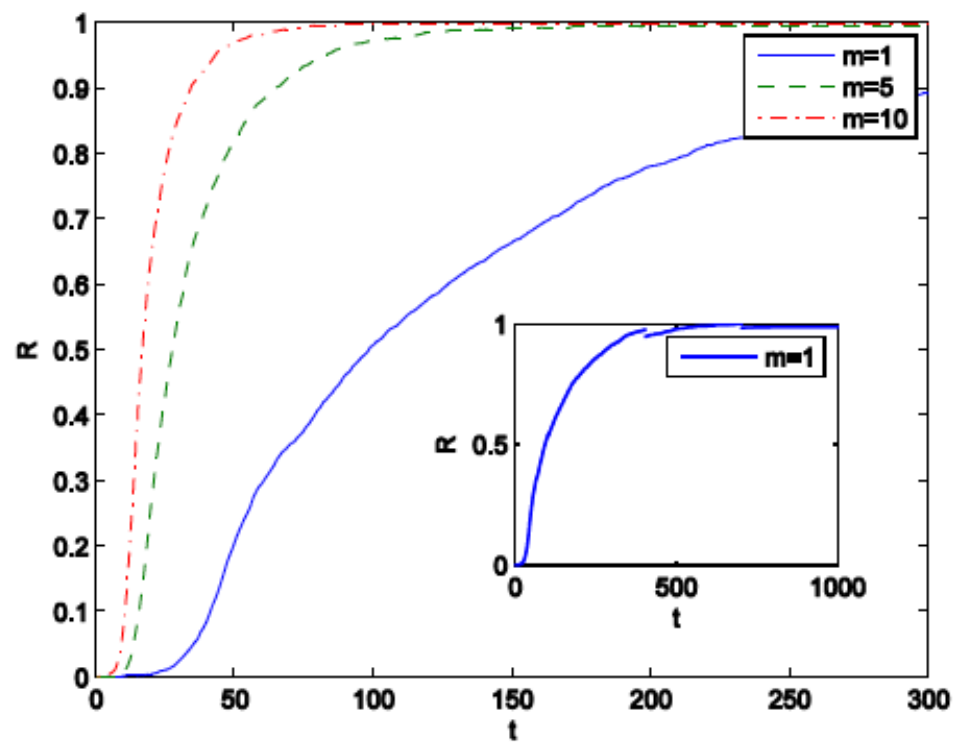

Fig. (4). Densities of stiflers over time with different information packet transmission quantity in homogeneous network.

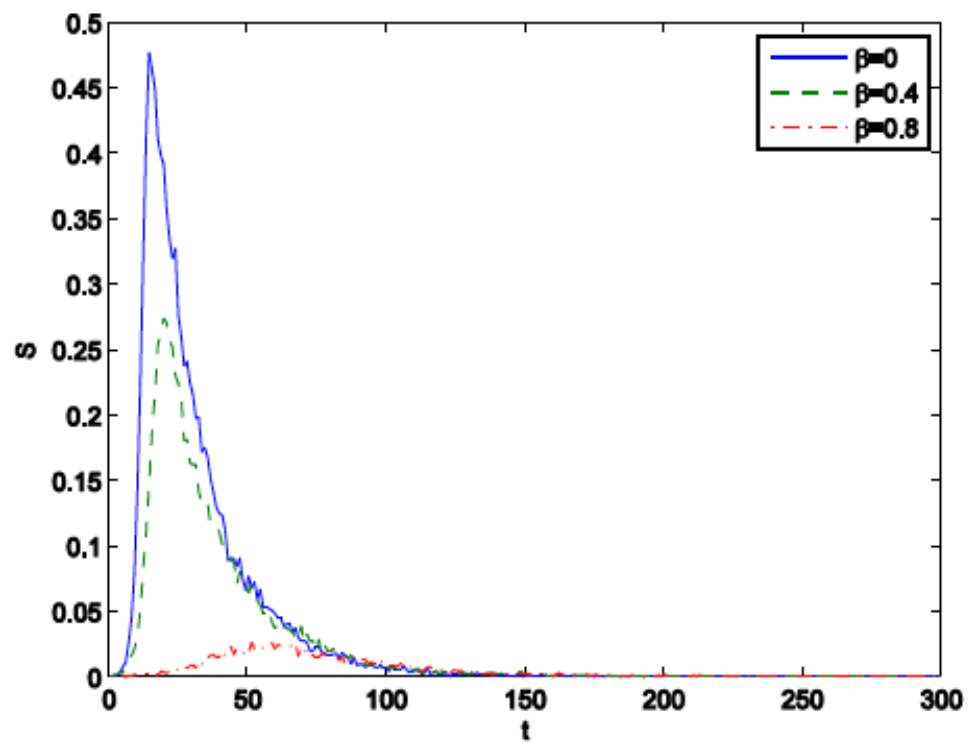

Fig. (5). Densities of spreaders over time with different refusing rate in homogeneous network.

information transmission of a node, and when a node transmission a few information to its neighbors at one step, it will last a long time before the densities of stiflers saturated. From the real world, we can explain this phenomenon as follows:

When a spreader sends more messages to acquaintance through E-mail or cell phone, almost all his friends could hear the rumor sooner, when they believe the rumor, they would spread it and make more people become spreaders. Because of more information exchange in network, a spreader no longer spread a rumor when they know the rumor is outdated or wrong. So the densities of spreader reach peak fast and rapid declines after that.

Because ignorants can get a few information from others, so they hardly hear of rumor, they would maintain the status until receive information packets from spreader. On the other hand, while an ignorant get information from spreader, many of them would like to believe the rumor, as time goes by, they may lose interests and become refractory, but it take a long time.

Next, we study the influence of refusing rate under given information transmission rate. We fix the parameters $m=5, \lambda=0.8, \alpha=0.3, \delta=0.6, \xi=0.5, \eta=0.5$. Figs. (5 and 6) illustrate how the densities of spreaders and stiflers change over time for different refusing rate with $\beta=0,0.4$ and 0.8 , respectively.

Fig. (5) shows that if an individual get limited information from his neighbors, as the refusing rate increase, more people do not believe rumor. Once they hear rumor, most of them will become stiflers directly. So the maximum of the spreader are smaller. Furthermore, while an individual sends the same number of information packages to his neighbors, the densities of spreaders is almost to zero at the same time. 


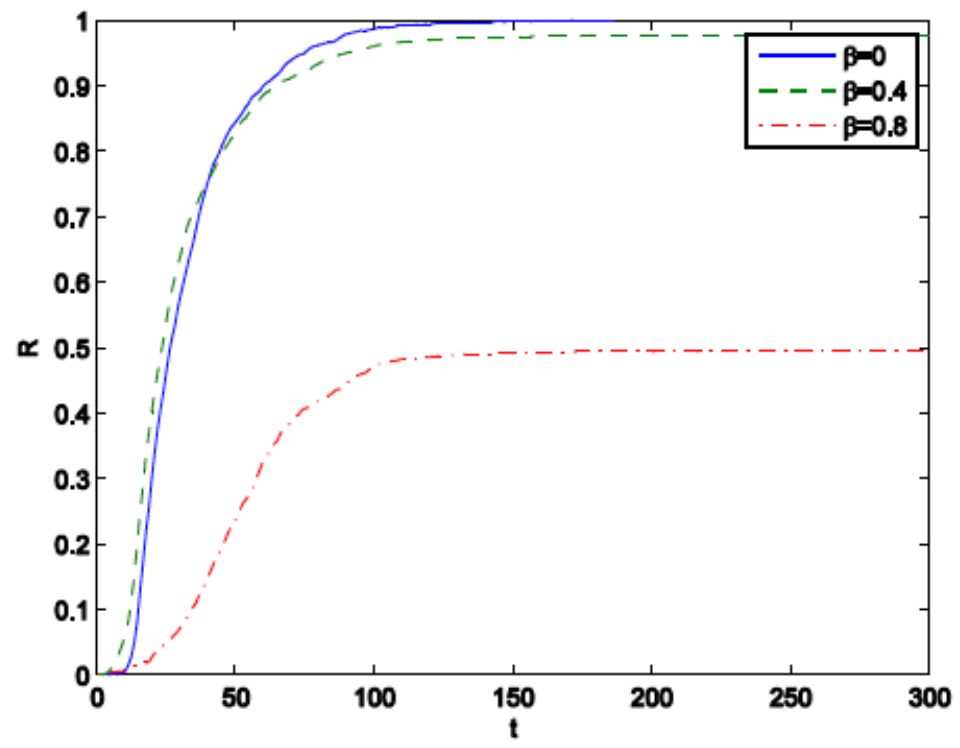

Fig. (6). Densities of stiflers over time with different refusing rate in homogeneous network.

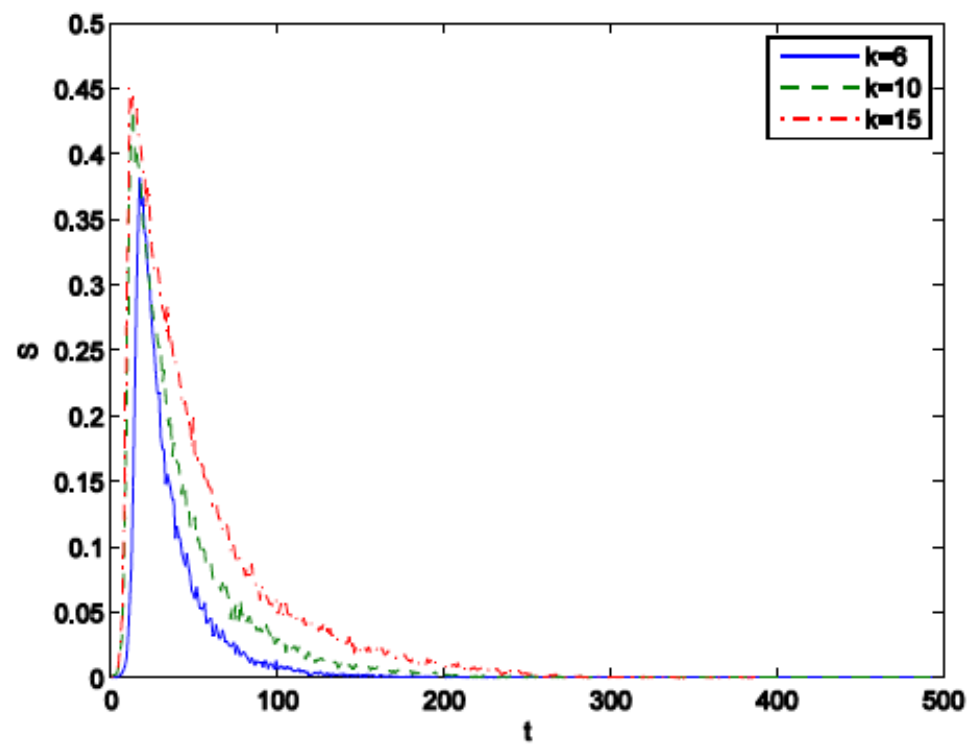

Fig. (7). Densities of spreaders over time with different average degree in homogeneous network.

Fig. (6) describes that as the refusing rate increase, the final density of stiflers become smaller. Because many people won't disseminate rumor, so some ignorants can not receive any messages about rumor and they will maintain the original state.

Finally, we study how the average degree of a node influences the rumor under limited information exchange. We fix theparameters From the Figs. (7 and 8). we can get the following result.

With the neighbor's increase of a node, the rumor propagated faster. And it will postpone the time of the spreader' final disappearance. The reasons is that when a person sends the same number of messages to his friends at each time step, as his friends become much, one may prefer to transmission not many but a message to a person. Under given information packet transmission quantity $m$, a node may send messages to his friends as much as possible at one time step. Therefore, the ignorant may become the spreader sooner. Moreover, if a person has many friends, but only a few of them transmission messages to him, when he becomes spreader, he may believe the rumor persisted for a longer time.

\section{RUMOR PROPAGATION IN INHOMOGENEOUS}

In this section, we numerically investigate the limited information transmission influence the rumor spreading in inhomogeneous network [23]. We use scale-free networks with the power-law degree distribution $P(k)=2 n^{2} k^{-3}$, where is the minimum node degree and the average connectivity of the network is $\langle k\rangle=2 n$. Here, we let $n=3$. The network size is fixed to be $N=2000$. In the initial condition, there is only one spreader in the network, and the others are ignorants. 


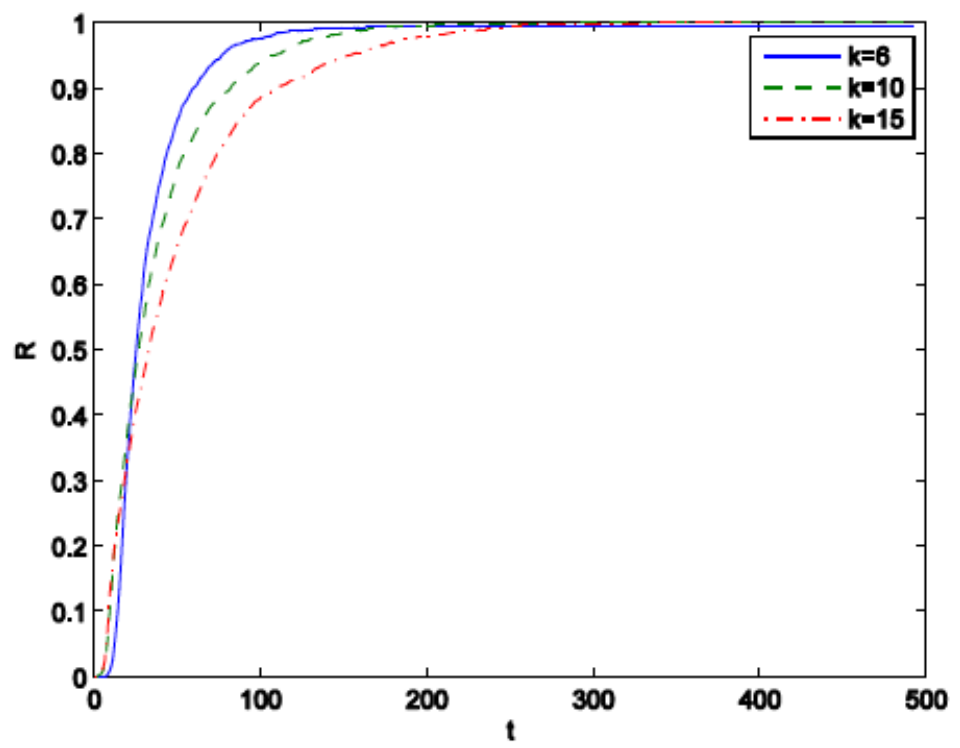

Fig. (8). Densities of stiflers over time with different average degree in homogeneous network.

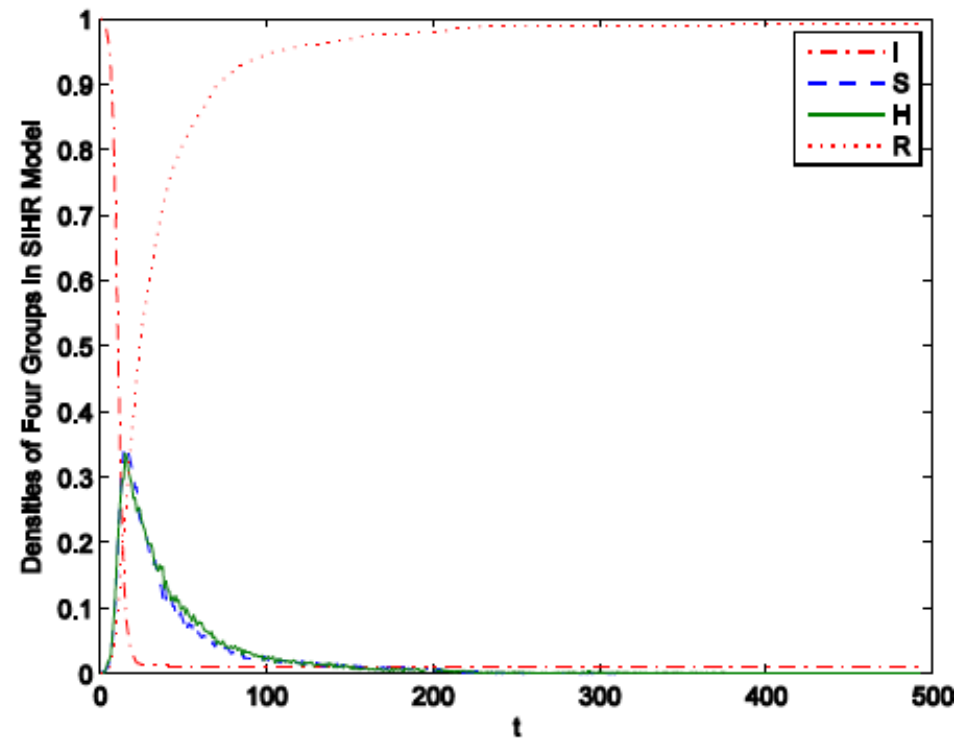

Fig. (9). Densities of Four Groups in inhomogeneous network.

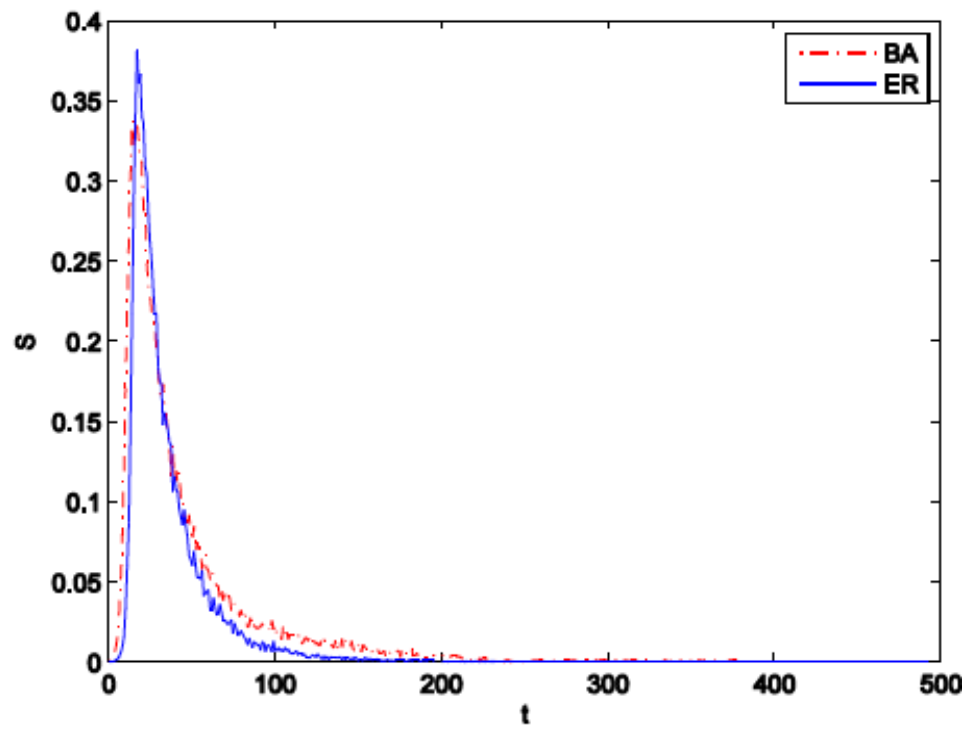

Fig. (10). Densities of spreaders over time inboth BA and ER network. 


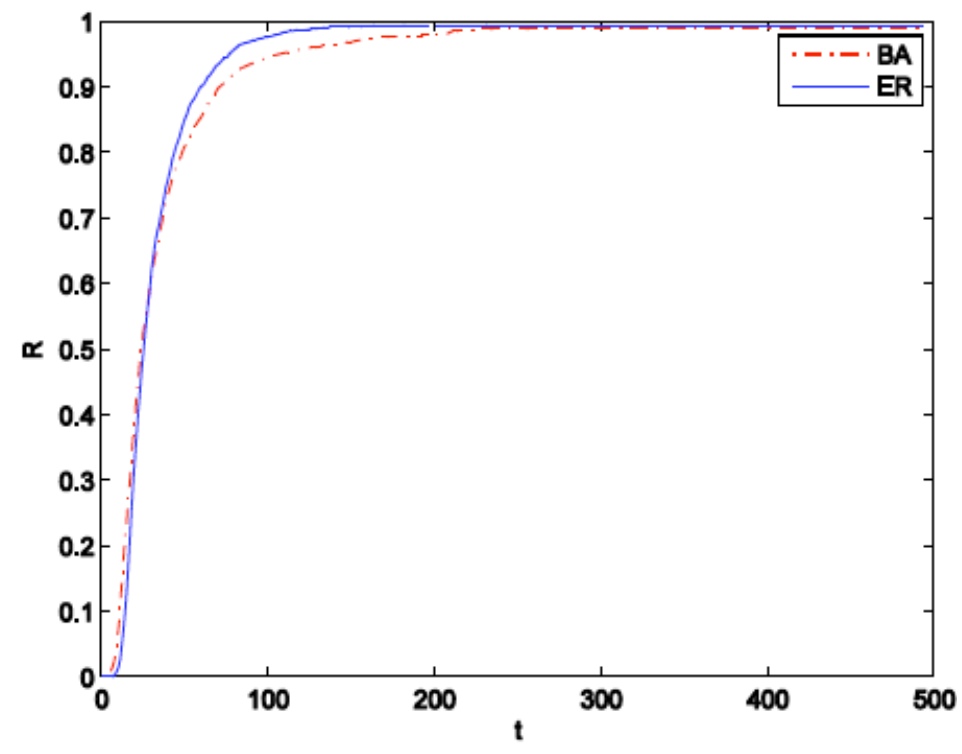

Fig. (11). Densities of stiflers over time in both BA and ER network.

We fix the parameters $m=5, \lambda=0.8, \beta=0.2, \alpha=0.3$, $\delta=0.6, \xi=0.5, \eta=0.5$. Fig. (9). illustrates the densities of the four groups in different state as a function of time, where a node could get information from all its neighbors. We can easily see that Fig. (9) is similar to Fig. (2) of the ER network but with some differences. Next we will compare the evolution all kinds of people between ER and BA network under limited information exchange.

Let the parameters $m=5, \lambda=0.8, \beta=0.2, \alpha=0.3$, $\delta=0.6, \xi=0.5, \eta=0.5$. As can be seen in Figs. (10 and 11), the rumor spreads faster in BA network than in ER network. For heterogeneous networks, there are high degree nodes which make information packets delivery more efficient. On other words, one may easily hear rumor in heterogeneous networks. Although the high degree nodes can easily become spreader, they turn into stiflers faster. Therefore, the maximum rumor influence in ER network bigger than in BA network become spreader, they turn into stiflers faster. Therefore, the maximum rumor influence in ER network bigger than in BA network.

\section{CONCLUSION}

The aim of this paper is to propose a new rumor propagation model under limited information exchange. In real life, an individual cannot get all of his friends' information; let put it another way, one may not transmit massages to all his acquaintances at once. Therefore, our model is more realistic.

According to the simulation and our analysis, we find that if people send a few messages to others, the rumor may be disseminated for a long time, and if one would like to send more information to his friends, the rumors will disappear sooner. Moreover, improving people's accomplishment is an effective method to curb the rumor. Additional, under given information packet transmission quantity of every time for each node, while an individual have more neighbors, it will postpone the time of the spreaders' final disappearance.
At last, based on the comparison the limited information transmission influence the rumor spreading between in ER network and in BA network, we find that the maximum rumor influence in homogeneous network bigger than in inhomogeneous network.

\section{CONFLICT OF INTEREST}

The authors confirm that this article content has no conflict of interest.

\section{ACKNOWLEDGEMENTS}

This research is supported by National Natural Science Fund (71102160), Humanities and Social Sciences Project of Ministry of Education (10YJC630281), China Postdoctoral Science Foundation funded project (2012T50471) \& Advanced Talent Project of Jiangsu University (09JDG050).

\section{REFERENCES}

[1] S. Galam, "Modeling rumors: the no plane pentagonfrench hoax case", Physica A Statist. Mechan. Appl., vol. 320, pp. 571-580, 2003.

[2] R. Bhavnani, M.G. Findley, and J. H. Kuklinski, "Rumor dynamics in ethnic violence", J. Polit., vol. 73, pp. 876-892, July 2009.

[3] M. Kosfeld, "Rumours and markets ", J. Math. Econ. Elsevier, vol. 41, no. 6, pp. 646-664, Sep. 2005.

[4] A. J. Kimmel, "Editorial Commentary: Rumors and the Financial Marketplace", J. Behav. Finance, vol. 5, pp.134-141, 2004.

[5] D. J. Daley, and D. G. Kendall, "Epidemics and rumors", Nature, vol. 1, pp. 42-55, 1965

[6] D. J. Daley, and J. Gani, "Epidemics Modelling: An Introduction", Cambridge University Press: Cambridge, UK, 1999, pp.88-102.

[7] J. Zhou, Z. H. Liu, and B. W. Li, "Influence of network structure on rumor propagation", Phys. Lett. A, vol. 368, pp. 458-463, 2007.

[8] F. Chierichetti, S. Lattanzi, and A. Panconesi, "Rumor spreading in social networks", Theor. Comput. Sci., vol. 412, pp. 2602-2610, Nov. 2011.

[9] L. J. Zhao, Q. Wang, J. J. Cheng, D. Zhang, T. Ma, Y. C. Chen, and J.J. Wang, "The impact of authorities' media and rumor dissemination on the evolution of emergency", Physica A: Statist. Mechan. Appl., vol. 391, no. 15, pp. 3978-3987, 2009.

[10] L. Huo, J. Lan, Z. Wang, "New parametric prioritization methods for an analytical hierarchy process based on a pairwise comparison matrix", Math. Comput. Modell., vol. 54, pp. 2736-2749, Dec. 2011. 
[11] N. DiFonzo, M. J. Bourgeois, J. Suls, C. Homan, N. Stupak, B. P. Brooks, D. S. Ross, and P. Bordia, "Rumor clustering, consensus, and polarization: Dynamic social impact and self-organization of hearsay", J. Experim. Soc. Psychol., vol. 49, pp. 378-399, 2013.

[12] M. Nekovee, Y. Moreno, G. Bianconi, and M. Marsili, "Theory of rumour spreading in complex social networks", CoRR, abs/0807, vol. $1,2008$.

[13] B. Doerr, A. Huber, and A. Levavi, "Strong robustness of randomized rumor spreading protocols", Discrete Appl. Math., vol. 161, pp. 778-793, 2013

[14] M. Yamir, N. Maziar, and A. F. Pacheco, "Dynamics of rumor spreading in complex networks", Phy. Rev. E (Statist., Nonlinear, Soft Mat. Phys.), vol. 69, pp. 69-75, 2004.

[15] J. Gu, W. Li, and X. Cai, "The effect of the forget-remember mechanism on spreading", Eur. Phys. J. B, vol. 62, pp. 247-255, Apr. 2008.

[16] J. R. C. Piqueira, "Rumor propagation model: an equilibrium study", Math. Probl. Eng., vol. 2010, no. 2010, pp.1-7, 2010.

[17] Z. Zhang, and Z. Zhang, "An interplay model for rumour spreading and emergency development", Physica A: Statist. Mechan. Appl., vol. 388, pp. 4159-4166, June 2009.

[18] Z. Liu, Y. Lai, and N. Ye, "Propagation and immunization of infection on general networks with both homogeneous and heterogeneous components", Phys, vol. 67, pp. 1-15, Mar. 2003.

[19] L. Zhao, Q. Wang, J. Cheng, Y. Chen, J. Wang, and W. Huang, "Rumor spreading model with consideration of forgetting mechanism: A case of online blogging Live-Journal", Physica A: Statist. Mech. Appl., vol. 390, pp. 2619-2626, 2009.

[20] L. Zhao, Q. Wang, J. Cheng, D. Zhang, T. Ma, and Y. Chen, "The impact of authorities media and rumor dissemination on the evolution of emergency", Physica A: Statist. Mech. Appl., vol. 391, pp. 3978-3987, 2012

[21] R. Albert, and A. L. Barabasi, "Statistical mechanics of complex networks", Rev. Mod. Phys, vol. 74, pp. 47-97, 2002.

[22] Z. Liu, Y. Lai, N. Ye, and P. Dasgupta, "Connectivity distribution and attack tolerance of general networks with both preferential and random attachments", Phys. Lett. A, vol. 303, pp. 337-344, 2002.

[23] C. M. Schneider, T. Mihaljev, S. Havlin and H. J. Herrmann, "Suppressing epidemics with a limited amount of immunization units", Phys. Rev. E, vol. 84, p. 061911, 2011.

Received: September 22, 2014

Revised: November 30, 2014

Accepted: December 02, 2014

(C) Mengyun and Wenjuan; Licensee Bentham Open.

This is an open access article licensed under the terms of the Creative Commons Attribution Non-Commercial License (http://creativecommons.org/licenses/by-nc/3.0/) which permits unrestricted, non-commercial use, distribution and reproduction in any medium, provided the work is properly cited. 